TIPA. Travaux interdisciplinaires sur la parole et le langage

$27 \mid 2008$

Apprentissage et acquisition d'une langue seconde

\title{
Premiers énoncés d'une enfant bilingue précoce français-finnois
}

The first sentences produced by a simultaneous French-Finnish bilingual child

\author{
Aïno Niklas-Salminen
}

\section{OpenEdition}

1 Journals

Édition électronique

URL : https://journals.openedition.org/tipa/309

DOI : $10.4000 /$ tipa.309

ISSN : 2264-7082

Éditeur

Laboratoire Parole et Langage

Édition imprimée

Date de publication : 1 juin 2008

Pagination : $93-110$

ISSN : 1621-0360

Référence électronique

Aïno Niklas-Salminen, «Premiers énoncés d'une enfant bilingue précoce français-finnois », TIPA.

Travaux interdisciplinaires sur la parole et le langage [En ligne], 27 | 2008, mis en ligne le 22 janvier 2013, consulté le 08 septembre 2021. URL : http://journals.openedition.org/tipa/309; DOI : https://doi.org/ 10.4000/tipa.309

Ce document a été généré automatiquement le 8 septembre 2021.

\section{cc) (†) $९$}

La revue TIPA. Travaux interdisciplinaires sur la parole et le langage est mise à disposition selon les termes de la licence Creative Commons Attribution - Pas d'Utilisation Commerciale - Pas de Modification 4.0 International. 


\title{
Premiers énoncés d'une enfant bilingue précoce français-finnois
}

The first sentences produced by a simultaneous French-Finnish bilingual child

\author{
Aïno Niklas-Salminen
}

1 On dit généralement que l'enfant commence à parler lorsqu'il se met à produire des mots de la langue de son environnement. L'acquisition du lexique apparaît comme l'un des processus fondamentaux qui participent à l'émergence du langage. L'enfant bilingue précoce simultané ${ }^{1}$ est en contact avec deux langues au moment où il apprend à parler. Sa tâche est plus complexe que celle de l'enfant monolingue parce qu'il se trouve confronté à un nombre plus grand de signaux acoustiques différents, sans savoir automatiquement à quelle langue ils appartiennent, ni même qu'ils appartiennent à des langues différentes. De plus, il doit acquérir rapidement la faculté de reconnaître que le système de parole est arbitraire. Différencier les langues, éviter les interférences, apprendre à catégoriser les entrées acoustiques en deux systèmes contrastés sont des tâches réservées au bilingue. Et pourtant le bilingue précoce peut acquérir deux langues simultanément, sans prendre deux fois plus de temps que le monolingue.

\section{L'enfant observée}

2 Nous proposons d'observer les premiers énoncés produits par une fille bilingue précoce (désignée par M. dans le texte) qui a développé les deux langues, le français et le finnois, dès le début de l'acquisition du langage avec son développement général, dans un contexte d'apprentissage informel. Il s'agit d'une enfant unique. D'après les études de certains chercheurs (Deprez, 1994, p. 76), un couple avec un seul enfant réussit souvent à maintenir un comportement stable au niveau des deux langues. Dans les familles avec plusieurs enfants, on peut parfois noter une différence entre les aînés et leurs cadets. Les premiers semblent avoir plus de facilités à manier les deux langues que les seconds. 
3 M. est une bilingue biculturelle. Les deux langues et les deux cultures sont valorisées par son entourage. Elle s'identifie positivement aux groupes auxquels elle appartient. Elle vit avec ses parents en France. Sa mère est bilingue tardive finnophonefrancophone, son père est francophone et ne connaît que quelques mots en finnois. Ses parents ont essayé de suivre avec rigueur le principe « une personne - une langue » qui a permis de susciter chez elle une association automatique entre une langue et une personne de son entourage.

4 Le bilingue précoce a un comportement langagier différent de celui du bilingue tardif. Dans l'acquisition du langage en général, la "période critique » pour les capacités langagières est définie comme la période pendant laquelle l'enfant possède des capacités optimales pour l'acquisition du langage en rapport avec la maturation du système nerveux central. Lenneberg (1967) situe l'existence de cette période entre 2 ans et la puberté. Johnson et Newport (1989) considèrent qu'un déclin dans les compétences linguistiques se produit déjà bien avant la puberté, entre 6 et 8 ans. Ces chercheurs suggèrent que, contrairement à l'acquisition de la langue maternelle qui repose sur des mécanismes innés et se fait automatiquement, sans effort, l'acquisition de la seconde langue après la fin de la période critique, est un processus laborieux, reposant sur des mécanismes conscients.

5 On peut se demander si les deux langues d'un bilingue sont traitées par les mêmes aires cérébrales, ou bien par des aires partiellement ou totalement distinctes. Les avancées technologiques de ces dernières années permettent de mieux comprendre le fonctionnement du cerveau (Pallier \& Argenti, 2003, Pallier, 2006). Même si l'imagerie par résonance magnétique du bilinguisme en est encore à ses débuts, un résultat important peut d'ores et déjà être considéré comme établi : plus une seconde langue a été acquise jeune et/ou est bien maîtrisée, plus les activations dues à L1 et L2 sont similaires. Les aires qui sous-tendent les traitements en L1 et L2 se recouvrent presque parfaitement chez les bilingues de haut niveau. Ce résultat n'a rien d'évident. En effet, avant l'avènement de l'imagerie cérébrale, on aurait pu s'attendre, au contraire, à ce que deux langues bien maîtrisées soient sous-tendues par des aires cérébrales distinctes puisqu'au niveau comportemental elles interfèrent peu entre elles (Kim et al., 1997, p. 171-174).

6 En ce qui concerne les bilingues «non parfaits », c'est-à-dire sans doute la majorité des bilingues, les zones activées par L2 semblent être plus variables d'un individu à l'autre et ne semblent recouvrir que partiellement celles de L1. Une question actuellement non résolue est de savoir si les aires activées par la seconde langue sont véritablement spécifiques à celle-ci, ou bien si, dans certaines circonstances, elles pourraient aussi traiter la première langue. Par exemple, travailler avec une seconde langue moins bien maîtrisée que la première peut demander plus d'efforts ou d'attention, ce qui entrâne typiquement des augmentations de l'activité cérébrale.

7 Il est passionnant d'observer les pratiques langagières d'une enfant bilingue précoce qui parle deux langues très éloignées l'une de l'autre. Le finnois, qui est parlé par cinq millions de locuteurs, est la seconde langue finno-ougrienne après le hongrois. Il fait partie du sous-groupe dit fennique avec l'estonien et plusieurs langues en voie de disparition: live, vote, ingrien, vepse. Son écriture phonétique, ses quinze cas de déclinaison, ses suffixes possessifs, ses particules enclitiques, son auxiliaire de négation, entre autres, sont des traits qui surprennent souvent les locuteurs habitués aux langues indo-européennes. 
8 L'enregistrement et la prise de notes d'un grand nombre d'énoncés produits par M. ont servi de support à cette étude. Un vaste corpus varié, authentique et précis a été recueilli. La transcription orthographique a été choisie pour la transcription des énoncés car la transcription phonétique aurait été difficile à lire et la prononciation effective n'aurait eu aucune incidence sur les analyses. Cependant la transcription phonétique a été indiquée entre crochets chaque fois que cela paraissait nécessaire. La transcription orthographique est évidemment déjà une interprétation de la langue et implique tout un ensemble de règles (découpage en morphèmes, accord, etc.) que l'enfant ne maitrise pas encore.

\section{Les énoncés à un mot}

9 La production des sons, qui commence dès les premières semaines de la vie, prend tout son intérêt pour l'étude du développement de la parole lorsque l'enfant arrive à maitriser sa phonation, vers 5 mois, et lorsque s'instaure un contrôle articulatoire au cours de la deuxième moitié de la première année. Le babillage, c'est-à-dire la production de syllabes conformes à celles qui fondent la structure phonologique des langues, est la première étape du parcours jusqu'au développement phonologique propre à la production de mots et de phrases.

10 L'enfant produit d'abord des sons articulés analogues à ceux utilisés dans les langues naturelles. Il acquiert ensuite l'organisation phonologique propre à sa langue maternelle. Il lie enfin les schémas sonores qu'il entend et produit aux représentations qu'il a des objets, des actions, des événements, et organise ses connaissances linguistiques en fonction des principes grammaticaux de sa langue (Bertoncini \& Boysson-Bardies, 2003, p. 114).

11 Même si l'acquisition du lexique est un processus long, les deuxième et troisième années d'existence de l'enfant représentent la période cruciale de sa constitution. L'âge où apparaissent les premiers mots est assez variable, mais à partir de la fin de la première année, et pendant la première moitié de la deuxième année, l'enfant commence à produire des mots tout en continuant de babiller. Le développement du premier vocabulaire est lent, et 4 ou 5 mois sont en général nécessaires pour que l'enfant atteigne un vocabulaire de production de plus de 50 mots. Les premiers mots ont des formes simples, parfois très précises, mais d'une extrême variabilité. Pour Ferguson (1986) et MacKen (1979), au cours de cette période, l'unité de production serait le mot entier. L'enfant se réfère à une représentation incomplètement spécifiée, fournissant la forme globale, la métrique, quelques aspects saillants de l'articulation et la structure accentuelle des mots.

12 M. produit son premier mot vers un an. Son répertoire lexical s'accroît lentement pour atteindre une vingtaine de mots vers 15 mois. On assiste alors à une brusque extension du vocabulaire qui passe à environ 180 mots vers 19 mois. L'interprétation purement linguistique des énoncés de l'enfant n'est pas possible sans la connaissance du contexte situationnel. Il ne suffit pas de savoir ce que l'enfant dit, mais à qui il le dit, à propos de quoi, en présence de quels objets, en l'accompagnant de quels gestes, de quelles mimiques, de quelles réactions, avec quel résultat, etc.

13 Dans les paragraphes qui suivent, on s'intéressera d'abord aux mots français et aux mots finnois dans les énoncés produits par M. Il sera important de voir si les mots de 
l'une des deux langues sont plus employés par elle que les mots de l'autre langue. Ensuite, on examinera les parties du discours auxquelles les mots appartiennent, ainsi que les différents domaines d'expérience désignés par ces mots. Ces observations permettront de capter les similitudes et les différences au niveau des deux lexiques. On analysera également les synonymes bilingues trouvés dans les énoncés. En effet, à côté des mots français et des mots finnois qui possèdent chacun leur référent, il y a des mots en double, un mot français et un mot finnois pour désigner le même référent (papa/isä ; bébé/vauva).

\subsection{Les deux lexiques}

On peut d'abord diviser les unités produites par M. en mots français et en mots finnois. Il y a quelques mots qui peuvent faire partie des deux langues. Parmi ces mots " neutres ", on trouve des unités qui désignent le même référent dans les deux langues (légo/leego "pièce de plastique emboîtable d'un jeu de construction») et des unités homonymes qui désignent des référents différents (pipi fr. "urine »/fin. "douleur ", « souffrir », « cassé, e »).

Tableau 1. Quantité des mots français par rapport aux mots finnois

\begin{tabular}{|l|l|l|}
\hline Langue & Nombre de mots & Pourcentages \% \\
\hline Français & 105 & $38 \%$ \\
\hline Finnois & 173 & $62 \%$ \\
\hline
\end{tabular}

Tableau 2. Répartition des mots français et des mots finnois en fonction des parties du discours

\begin{tabular}{|l|l|l|}
\hline Nature des mots & Français & Finnois \\
\hline Substantifs & $66 \%$ & $75 \%$ \\
\hline Verbes & $15 \%$ & $12 \%$ \\
\hline Adjectifs & $6 \%$ & $6 \%$ \\
\hline Autres & $13 \%$ & $7 \%$ \\
\hline
\end{tabular}

Parmi les énoncés à un mot produits par M. jusqu'à l'âge de 1 an et 10 mois, on trouve 105 mots français et 173 mots finnois. Les mots finnois représentent donc $62 \%$ du vocabulaire global. Parmi les mots français, il y a $66 \%$ de substantifs ( $49 \%$ de noms communs, $17 \%$ de noms propres), $15 \%$ de verbes, $6 \%$ d'adjectifs et $13 \%$ de mots qui appartiennent aux autres parties du discours. Parmi les mots finnois, on note les mêmes tendances : $75 \%$ de substantifs (68 \% de noms communs, $7 \%$ de noms propres), $12 \%$ de verbes, $6 \%$ d'adjectifs, $7 \%$ de mots appartenant aux autres parties du discours.

On constate aussi que la plupart des mots dans les deux langues contiennent deux syllabes (fr. bateau $[1,6]^{2}$, câlin $[1,6]$; fin. hattu « chapeau » $[1,7]$, heppa « cheval » $\left.[1,10]\right)$. 
Il y a quelques mots à une syllabe (fr. chat $[1,6]$, clé $[1,7]$, fleur [1,6]; fin. kii «fermé " $[1,7])$ et quelques mots à trois syllabes (fr. [sãgile] (= sanglier) [2] ; fin. [aujonko] (= aurinko) « soleil » $[1,6]$, etana « escargot » $[1,10])$.

\subsubsection{Les différentes parties du discours}

17 On peut classer les mots selon les parties du discours auxquelles ils appartiennent. Il ne faut pas oublier que l'enfant n'emploie pas toujours les mots avec la même signification que les adultes. Chez l'enfant, un même mot peut désigner aussi bien un objet qu'une action, encore que le plus souvent, il renvoie plus vraisemblablement à une situation. Par exemple, le mot finnois pipi peut être un substantif («douleur»), un verbe (" souffrir ») ou un adjectif (« cassé »).

\section{Substantifs}

On vient de voir que les substantifs forment la partie du discours la plus fréquente dans les deux langues chez M. En français les substantifs ne sont pas déterminés. La plupart des substantifs finnois se trouvent au nominatif singulier : ankka « canard » $[1,6]$, possu " cochon" [1,7]. Il y a aussi quelques substantifs qui sont au partitif singulier ou pluriel : kalaa « du poisson» [1,7], kavja « du café » [1,7]. Un substantif est conjugué à l'inessif (laukussa « dans le sac ») et un autre à l'illatif (Suomeen « entrer en Finlande »).

\section{Verbes}

$1915 \%$ des mots français et $12 \%$ des mots finnois employés par M. dans ses énoncés à un mot sont des verbes. Plusieurs verbes français se trouvent à la deuxième personne du singulier et parfois à la deuxième personne du pluriel de l'impératif : attends! [1,6], regarde! [1,6], allez! [1,7]. Il y a aussi des verbes qui se trouvent à l'infinitif ou au participe passé : gâter $[1,6]$, partir $[1,6]$, moucher $[1,10]$.

20 Les verbes finnois employés par M. sont souvent conjugués à la deuxième personne du singulier ou à la première personne du pluriel de l'impératif : anna « donne » [1,6], kato «regarde» $[1,6]$, mennään «allons» $[1,6]$. Il y a aussi des verbes conjugués à la troisième personne du singulier de l'indicatif ou à l'infinitif : auttaa «aider » [1,7], polkee « pédaler » $[1,7]$.

\section{Adjectifs}

$216 \%$ des mots français et autant de mots finnois employés par M. dans ses énoncés à un mot sont des adjectifs. Ceux-ci se trouvent au masculin en français. Il n'y a pas de genre grammatical en finnois. La plupart des adjectifs sont qualificatifs dans les deux langues, il y a aussi un participe passé (fermé). Les adjectifs sont surtout non axiologiques : fr. chaud [1.6], froid [1.6], petit [1.6]; fin. pikku « petit » [1.6], auki « ouvert» [1.7], puna (= punainen) « rouge » [1.10].

22 Les adjectifs de couleur se trouvent seulement en finnois. On rencontre aussi des adjectifs axiologiques mélioratifs dans les deux langues : fr. beau [1.6], [loli] (=joli) [1.6] ; fin. hellä « tendre » [1.6], kiitti (= kiltti) « gentil» [1.10].

- Autres parties du discours 
$13 \%$ des mots français et $7 \%$ des mots finnois dans les énoncés à un mot de $\mathrm{M}$. appartiennent aux autres parties du discours. On y trouve des adverbes (fr. encore [1.6], alors [1.6], mal [1.6]; fin. äkkiä «vite» [1.7], miksi? "pourquoi?» [1.10]), des prépositions (fr. à côté [1.10]; fin. mukaan « avec » [1.10]), des pronoms (fr. quoi ! [1.6]; fin. tää « ceci » [1.6]) et des interjections (coucou [1.6], op laa [1.7], kili [1.6]).

\subsubsection{Les différents domaines d'expérience}

Plusieurs domaines d'expérience sont touchés par les référents des substantifs employés par M. En français et en finnois, il y a surtout des substantifs qui désignent des animaux, des êtres humains et de la nourriture. Les deux lexiques contiennent également des substantifs qui désignent des parties du corps et des choses liées à l'hygiène, des éléments de la nature, des besoins naturels et des jouets.

Les substantifs désignant des moyens de transport sont nettement plus courants en français qu'en finnois. En revanche, dans le lexique finnois de M., il y a beaucoup de mots qui désignent des choses liées à la maison, comme kello "montre, horloge ", lamppu « lampe ", patja « matelas ». Ces mots-là sont absents de son lexique français. Le lexique finnois contient aussi des mots qui désignent des vêtements et des bijoux. Ces mots-là sont très rares dans le lexique français. Les noms propres dans les deux langues sont des noms de personnes et d'animaux connus par l'enfant ainsi que des noms de personnages de bandes dessinées ou de livres pour enfants. Les verbes français et finnois dénotent surtout des actions et des mouvements. Les adjectifs désignent la plupart du temps un attribut relatif à la taille, une appréciation ou la dénomination d'une couleur.

\subsubsection{Les similitudes et les différences dans les deux lexiques}

On peut constater que les mots des deux lexiques présentent un certain nombre de similitudes. Les mots français et finnois employés par M. sont surtout bisyllabiques. On trouve à peu près les mêmes proportions de verbes et d'adjectifs dans les deux langues. Les substantifs sont nettement plus nombreux que les mots appartenant aux autres parties du discours.

On peut noter aussi quelques différences entre les deux lexiques de M. Les unes semblent être liées aux structures des langues, d'autres au contexte, à la situation. L'utilisation des adverbes est beaucoup plus fréquente en français qu'en finnois. En ce qui concerne les substantifs, on peut trouver des différences au niveau des domaines d'expérience. On a l'impression qu'il y a des mots liés au père français et des mots liés à la mère finlandaise. Ces mots semblent être directement en rapport avec les activités menées par les deux parents.

Les substantifs français ont toujours la même forme, les substantifs finnois changent parfois de forme suivant les cas de déclinaison. Parmi les premiers mots finnois de $\mathrm{M}$. certains se trouvent au partitif, à l'inessif ou à l'illatif. Le partitif peut désigner la partie d'un tout (maito-o « du lait », kukki- $a$ " des fleurs »), l'insessif la présence à l'intérieur d'un lieu clos (lauku-ssa « dans le sac ») et l'illatif le mouvement de pénétration dans un lieu clos (Suom-een « (entrant) en Finlande »).

Plusieurs facteurs structurels et sémantiques déterminent le choix de tel ou tel cas dans un énoncé. Par exemple, le cas du sujet ou de l'objet peut dépendre de la nature et du 
sens du verbe, du sens du mot qui fonctionne en tant qu'objet, ainsi que de la structure syntaxique de l'énoncé. Le sujet d'un verbe personnel peut être au nominatif ou au partitif. Il est au partitif s'il s'agit d'un nom non dénombrable employé avec une valeur partitive ; il l'est également s'il s'agit de nier ou de mettre en doute son existence. Le sujet peut se trouver aussi au génitif. Ce cas est obligatoire, par exemple, dans les constructions qui contiennent le verbe täytyy « devoir ».

Le complément d'objet peut se trouver, entre autres, à l'accusatif ou au partitif. Une différence est faite entre l'objet total, marqué par l'accusatif et l'objet partiel, exprimé par le partitif. Cette distinction paraît tout à fait logique avec les noms de matière, de qualité ainsi qu'avec les substantifs désignant des notions non dénombrables (maidon «le lait »/maitoa « du lait»). L'opposition accusatif/partitif peut également exprimer l'aspect résultatif ou non résultatif de l'action en général. L'objet total apparaît avec un verbe qui désigne une action touchant la totalité de l'objet et l'objet partiel avec un verbe qui ne concerne qu'une partie de cet objet. Ce qui est intéressant, c'est que le choix entre l'objet partiel et l'objet total dépend aussi bien du sens du verbe que de celui du mot qui exprime l'objet.

31 Les premières manifestations grammaticales commencent à apparaître dans les énoncés de l'enfant surtout au moment où il se met à combiner des mots entre eux. Même si l'enfant finnophone emploie des formes partitives, telles que maito-o et kavj-a, il ne s'agit pas vraiment de l'acquisition du cas partitif. Les premières occurrences des cas de déclinaison n'expriment pas encore les vraies valeurs des cas. L'enfant a acquis, par imitation, telle ou telle forme du mot fréquemment entendue dans la communication quotidienne.

D'après Toivanen (1993, p. 274) on peut parler de cas de déclinaison seulement à partir du moment où l'enfant commence à former des cas différents sur le même radical. Le partitif, l'inessif et l'illatif sont les premiers cas de déclinaison utilisés par M. L'acquisition des suffixes semble passer par plusieurs phases: l'imitation des suffixes (les premières apparitions des suffixes), la vraie utilisation occasionnelle du bon suffixe, les productions analogiques générales et erronées, la maitrise des règles même avec les formes irrégulières. $M$. acquiert vers 2 ans- 2 ans et demi la plus grande partie des formes du système casuel du finnois.

On a vu que M. utilise plus de mots en finnois qu'en français dans ses premiers énoncés. Cela paraît assez logique dans la mesure où elle passe plus de temps avec sa mère qu'avec son père. Les différences de taille observées dans les deux lexiques du jeune bilingue proviennent souvent des facteurs environnementaux ou sociolinguistiques. Le vocabulaire est sensible à la fréquence d'exposition et aux répétitions. Les différences dans la manière d'acquérir d'une langue en fonction de la structure de celle-ci ont peutêtre aussi un impact sur le premier lexique de l'enfant bilingue. Il ne possède vraisemblablement pas au même moment les mêmes catégories lexicales dans chacune de ses langues.

34 Le rapide développement du lexique constaté autour de 18 mois chez la plupart des enfants unilingues s'observe aussi chez les enfants bilingues. Mais les deux langues ne se développent pas au même rythme: quand le lexique s'enrichit dans une langue, il peut stagner dans l'autre. Ce retard est parfois rattrapé un peu plus tard, mais très souvent un déséquilibre persiste entre les deux lexiques.

L'enfant bilingue entend deux noms différents pour les mêmes référents, mais n'entend peut-être pas les deux noms aussi systématiquement qu'un enfant monolingue. 
L'enfant bilingue peut acquérir le mot qu'il entend en premier et commencer à l'employer dans les contextes des deux langues. Assez souvent, au processus d'acquisition est lié un fait émotionnel. C'est peut-être pour cela que l'enfant retient plus facilement ce mot-là. La forme du mot peut paraître amusante, il peut être associé à un autre mot ou il peut être plus facile à produire au niveau formel (mot plus court, plus facile à prononcer) que son équivalent dans l'autre langue (Leopold, 1949, p. 267 ; Vihman, 1985, p. 308-309).

\subsection{Les synonymes bilingues français-finnois} premières manifestations de la syntaxe dans le développement du langage. Ils apparaissent entre 18 et 24 mois, avec d'importantes variations individuelles. L'association de deux mots semble être universelle. On la remarque au même stade de développement chez des enfants locuteurs de langues différentes. L'enfant anglais, 
français, allemand, turc et hongrois, par exemple, commence par associer deux mots avant de faire des énoncés complexes. Ce stade des deux mots est vu plutôt comme représentant un progrès linguistique qu'un progrès conceptuel.

M. commence à produire des énoncés à deux mots à partir de 18 mois dont certains dans lesquels les deux mots se trouvent en français ou en finnois. On trouve aussi des énoncés hybrides qui contiennent un mot en français et un mot en finnois. Dans les paragraphes suivants, on observera d'abord la composition grammaticale des énoncés qui contiennent deux mots de la même langue. On mettra ensuite en relief les similitudes et les différences concernant les énoncés en français et ceux en finnois. À la fin, on examinera la composition des assemblages hybrides.

\subsection{Deux mots français}

42 Nous allons examiner d'abord la composition des énoncés où les deux mots sont en français puis les classer en fonction des parties du discours qui les composent.

\subsubsection{Les énoncés contenant un verbe}

La plupart des énoncés présentent des combinaisons substantif-verbe ou verbesubstantif $(20 \%)$. Dans la moitié des énoncés, le substantif précède le verbe, dans l'autre moitié, c'est le contraire. Le substantif n'est pas précédé d'un déterminant et le verbe se trouve au présent de l'indicatif (Mathilde marche [1.10]), au participe passé (maman partie [1.7]), à l'infinitif (chercher papa [1.10]) et même à l'impératif (papa viens ! [1.10] ; souffle maman! [1.11] ; tiens maman! [2.1]).

L'ordre des mots dans ces combinaisons sujet-verbe ou verbe-objet n'est pas encore stabilisé. Par exemple, dans parti bateau, le substantif bateau qui se trouve après le verbe peut être considéré comme étant le sujet de l'énoncé. Papa, dans l'énoncé chercher papa, est l'objet de l'énoncé. Maman, dans tiens maman, peut être considéré comme étant l'apostrophe de l'énoncé.

$11 \%$ des énoncés contiennent un pronom personnel de la deuxième ou troisième personne du singulier ( $i l, t u, m o i, t o i)$ et un verbe. La forme conjointe des pronoms personnels se trouve avant le verbe, la forme disjointe après : il travaille [1.10], balancer moi [2]. Quelques énoncés contiennent aussi la préposition à et un verbe : à boire [abwa] $[1.6]$.

\subsubsection{Les énoncés sans verbe}

$10 \%$ des énoncés contiennent une préposition (à, pour) et un substantif : à poils [1.6], pour maman [1.10] et $9 \%$ des énoncés sont constitués de deux substantifs : papa Raymond [1.10], pardon maman [1.11]. Il y a aussi quelques énoncés qui associent un substantif et un adjectif ou un adverbe: gros dodo [1.7], Magali méchante [2], caca vite [1.10]. Une grande partie des énoncés contient une partie de négation combinée avec un adjectif ou un adverbe : pas là [1.7], pas bon [1.10].

$4710 \%$ des énoncés à deux mots produits par M. contiennent des interjections qui peuvent être combinées entre elles ou avec un adjectif : oh la laa [1.6], oh joli [1.10]. On peut constater qu'une grande variété de combinaisons caractérise les énoncés dans lesquels les deux mots sont en français. On va voir, dans les paragraphes suivants, que 
les combinaisons de mots dans les énoncés à deux mots en finnois sont beaucoup moins variées.

\subsection{Deux mots finnois}

\subsubsection{Un substantif accompagné d'un adjectif}

$27 \%$ des énoncés de M. contenant deux mots finnois sont formés d'un substantif accompagné d'un adjectif. Ce dernier se trouve plus souvent avant le substantif qu'après : pikku potta " petit pot de chambre " [1.10], vauva iso "bébé gros » [1.10], lika nenä [1.11] «sale nez » [1.11], kylmää maitoo « du lait froid» [2]. Ces énoncés peuvent décrire la taille d'un objet ou d'un être, la qualité ou l'état d'un objet, l'âge d'un être et la couleur d'un objet. Les adjectifs mélioratifs (kiva «sympathique ", kiltti " gentil ", hassu «amusant») sont plus fréquents que les adjectifs péjoratifs (tumma = tuhma « méchant »).

Les substantifs et les adjectifs se trouvent presque exclusivement au nominatif. Il n'y a qu'un énoncé dans lequel les deux mots se trouvent au partitif (kylmää maitoo « du lait froid»).

\subsubsection{Deux substantifs}

50 Une grande partie des énoncés à deux mots en finnois $(22 \%)$ contiennent deux substantifs dont certains sont déclinés à l'illatif (kouluun, «à l'école», syliin « dans les bras») ou au génitif (isän "de papa»). On trouve des combinaisons de deux noms communs qui peuvent indiquer une possession (mummu hame "la jupe de mamie" [1.10], isän kiija «le livre de papa» [1.10]), une localisation (helmi kaulaa (= helmet kaulaan) «mettre les perles autour du cou» [1.10], Matilda kouluun « Mathilde (va) à l'école» [2]), une énumération (mummu ukki «mamie, papy» [1.10], kukko kana « un coq, une poule »[1.10]).

51 Une grande partie de ces énoncés forme des mots composés. Il y a des énoncés qui combinent un nom commun et un nom propre (Coco koira « Coco chien » [1.6], äiti Aïno «maman Aïno» [1.10], Vitsi-Matilda "plaisanterie Mathilde» [1.11]), deux noms communs (kissapaita « chatshirt » [1.11], likapeppu « saleté fesses » [2.1]).

Parmi les mots composés, on trouve un néologisme (iik-kiija « au secours livre, un livre qui fait peur »). Il est intéressant de constater que M. commence à déjà former des mots composés en finnois à 18 mois. Le finnois possède deux grands types de mots composés: les uns contiennent deux parties sémantiquement asymétriques (autotie, " autoroute»), les deux autres parties symétriques (parturi-kampaamo, "barbier/ coiffeur »). Il y a des mots composés figés et des mots composés occasionnels.

\subsubsection{Un substantif et un verbe}

Un grand nombre d'énoncés à deux mots en finnois produits par M. comporte un substantif et un verbe $(21 \%)$. Le substantif peut être le sujet (äiti suuttuu « Maman se fâche » [1.10], heppa keinuu «le cheval se balance » [1.10]) ou l'objet de l'énoncé (saadaan kaloja « on attrape des poissons » [1.10], juoda maitoo « boire du lait » [1.11]) de l'énoncé. L'ordre des mots n'est pas encore stabilisé. On constate que c'est souvent le substantif qui précède le verbe, même s'il fonctionne en tant qu'objet de l'énoncé. Les 
combinaisons Matilda piirtää « Mathilde dessine » et piirtää Matilda " dessine Mathilde » signifient la même chose. Dans les énoncés piirtää estenta = elefantti « dessine éléphant » et krokotiili piirtää « crocodile dessine », le substantif estenta « éléphant ", qui se trouve après le verbe, et krokotiili «crocodile ", situé avant le verbe, sont tous les deux des objets.

\subsubsection{Autres combinaisons}

54 Parfois les énoncés à deux mots en finnois combinent un substantif avec un adverbe (sukka pois «chaussette ailleurs» [1.10], tele pois " télé ailleurs » [2]), deux adjectifs (kiltti - tumma (= tuhma) "gentil - méchant » [2], inki (= kiinni) - auki «fermé, ouvert » [1.11]) ou une préposition avec un substantif (akanssa äiti (= äidin kanssa) "avec maman »[2]).

\subsubsection{Le français et le finnois : similitudes et différences}

55 Les énoncés français présentent plus de combinaisons différentes que les énoncés finnois. Le seul point commun dans les deux langues semble se trouver au niveau des énoncés associant un substantif et un verbe. Cette combinaison représente à peu près $20 \%$ des énoncés dans les deux langues. Le substantif peut être le sujet, l'objet ou le complément des énoncés.

Tableau 3. Composition des énoncés à deux mots dans les deux langues

\begin{tabular}{|l|l|l|}
\hline Nature des mots & Français & Finnois \\
\hline Subst. + v. & $20 \%$ & $21 \%$ \\
\hline Pronom + v. & $13 \%$ & $0 \%$ \\
\hline Prép. + subst. & $10 \%$ & $0,5 \%$ \\
\hline Subst. + subst. & $9 \%$ & $25 \%$ \\
\hline Interj. + interj. & $6 \%$ & $1,5 \%$ \\
\hline Subst. + adj. & $5 \%$ & $30 \%$ \\
\hline Interj. + adj./adv. & $5 \%$ & $3 \%$ \\
\hline Nég. + adj./adv. & $9 \%$ & $0 \%$ \\
\hline Prép. + v. & $6 \%$ & $0 \%$ \\
\hline Subst. + adv. & $7 \%$ & $10 \%$ \\
\hline Adj. + adj. & $5 \%$ & $6 \%$ \\
\hline Adv. + adj. & $5 \%$ & $3 \%$ \\
\hline
\end{tabular}


Ce tableau révèle quelques différences assez frappantes entre les énoncés en français et ceux en finnois. On constate, par exemple, qu'en français les énoncés qui combinent un pronom et un verbe sont relativement courants (13\%). Ces combinaisons ne se trouvent pas encore dans les énoncés en finnois. En français on trouve aussi des énoncés qui associent une préposition et un substantif. Ces combinaisons sont presque inexistantes en finnois. Les énoncés qui contiennent une partie de négation n'existent qu'en français. En revanche, les énoncés qui combinent un substantif et un adjectif sont très fréquents en finnois, mais relativement rares en français. Les associations de deux substantifs apparaissent souvent en finnois, mais rarement en français. analogues : le désir, la possession, la localisation, la qualité. Ces catégories peuvent être distinguées : celles qui associent deux mots pleins qui, pris séparément, ont un sens défini (chercher papa) et celles qui combinent un mot plein et un autre mot qui opère sur le premier. Par exemple, dans l'énoncé encore gâteau, le mot encore a valeur d'opérateur. Ces mots «fonctions » donnent naissance aux particules, telles que les prépositions, les démonstratifs, les quantificateurs ou les conjugaisons des verbes.

\subsection{Les énoncés hybrides}

Depuis quelques années, l'attention croissante à l'égard des faits de contacts entre langues a conduit les chercheurs à reconnaître dans les mélanges de langues un phénomène important. Auparavant on avait tendance à adopter une attitude critique envers ces combinaisons hybrides qui étaient associées aux représentations sociales de l'impur.

59 Actuellement on pense plutôt que ces fusions sont pleines d'intérêt pour tout ce qu'elles révèlent sur les aptitudes des individus capables de passer rapidement d'un code à l'autre. Si les individus mélangent les langues, ils le font souvent sur la base d'un choix délibéré. Le bilingue semble posséder une double compétence qui s'accroît au fur et à mesure de l'apprentissage. Cette forme particulière de parler se caractérise par un choix de langue plus variable, constamment renégociable et renégocié et/ou par des marques transcodiques de tout genre et notamment par le fait de passer rapidement à l'autre langue pour la durée d'un mot, d'une expression, d'une phrase, pour revenir ensuite à la langue de base (Lüdi, 1999, p. 13).

Les chercheurs attestent que les deux langues du bilingue précoce s'influencent mutuellement au cours du processus d'acquisition. Tous les spécialistes du bilinguisme ont trouvé des mélanges de langues dans les énoncés des enfants étudiés. Leur quantité semble varier en fonction de plusieurs critères: l'âge et les caractéristiques personnelles de l'enfant, la nature des langues acquises, les facteurs psycho- et sociolinguistiques, ainsi que les méthodes d'analyse (Genesee, 1995, p. 612, 619, 626).

61 Les mélanges de langues commencent à apparaître chez $\mathrm{M}$. au moment où elle se met à produire des énoncés à deux mots. On trouve quelques combinaisons hybrides déjà à l'âge de 1 an et 7 mois (encore hellä), mais la plupart de ces constructions deviennent fréquentes à l'âge de 1 an et 10 mois. 
Tableau 4. Composition des énoncés hybrides

\begin{tabular}{|l|l|}
\hline Substantif + substantif & $32 \%$ \\
\hline Substantif + verbe & $26 \%$ \\
\hline Substantif + adjectif & $13 \%$ \\
\hline Adv. de négation + substantif & $7 \%$ \\
\hline Adjectif + adjectif & $4 \%$ \\
\hline Pronom + verbe & $3 \%$ \\
\hline Adverbe + adjectif & $3 \%$ \\
\hline Substantif + adverbe & $3 \%$ \\
\hline Verbe + verbe & $3 \%$ \\
\hline Préposition + substantif & $2 \%$ \\
\hline Préposition + verbe & $1 \%$ \\
\hline Adverbe + adverbe & $1 \%$ \\
\hline Déterminant + substantif & $1 \%$ \\
\hline Pronom + pronom & $1 \%$ \\
\hline
\end{tabular}

62 Il est très intéressant de constater que la plupart des énoncés hybrides à deux mots produits par M. contiennent des doublets. Dans le même énoncé, elle emploie un mot dans une langue et son équivalent synonymique dans l'autre. Les doublets touchent essentiellement les substantifs (käsi main [1.10], isä papa [1.10], maitoo du lait [1.10]), mais aussi les adjectifs (märkä mouillé [1.10]), les verbes (assis istuu [1.10]) et parfois même les adverbes (äkkiä vite [1.10]) et les pronoms ([lot] (= l'autre) toine [1.10]).

63 M. associe souvent aussi un substantif en français à un verbe en finnois (bonbon hakee " chercher » [1.10], auttaa " aider » maman [1.10], poupée suuttuu « se fâche » [2]), ou, vice versa, un substantif en finnois à un verbe en français (laukku « le sac » tombé [1.10], pöly « la poussière » parti [1.10]). Le substantif peut être le sujet ou l'objet de l'énoncé.

64 Le substantif peut être en finnois et l'adjectif en français (setä « tonton » gentil [2.1]), ou, vice versa, le substantif en français et l'adjectif en finnois (iso « grande » moto [1.10]). On peut trouver aussi un pronom dans une langue, combiné avec un verbe dans l'autre langue (kaaduu «tomber » moi [2]) ou un adverbe dans une langue, combiné avec un adjectif dans l'autre, un substantif et un adverbe, etc. 


\section{Conclusion} sûrement plus difficiles à manier que les prépositions en français, dans la mesure où elles se déclinent suivant les cas. Par exemple, la postposition edessä « devant » connaît six formes différentes : edessä (inessif), edestä (elatif), eteen (illatif), edellä (adessif), edelle (ablatif), edeltä (allatif). Les prépositions et les postpositions sont aussi complétées par des suffixes possessifs : edessäni «devant moi», edessäsi «devant toi», edessämme « devant nous », edessänne " devant vous », etc.

Les premiers énoncés à un terme de M. contiennent soit un mot français, soit un mot finnois. Même si les deux lexiques présentent un certain nombre de similitudes, on peut noter quelques différences dont les unes semblent être liées aux structures des langues et les autres à la situation. Parmi les premiers mots finnois de M., quelques-uns changent de forme en fonction des cas de déclinaison. Parmi les énoncés à deux termes produits par M., on trouve des combinaisons de deux mots en français et des combinaisons de deux mots en finnois. Les assemblages hybrides permettent de découvrir la nature des premiers mélanges de langues. grammaticaux de la langue et les règles qui président à leur ordre et, d'autre part, déterminer le sens de ces structures. Pour parvenir au contrôle des morphèmes grammaticaux, l'enfant doit maîtriser trois sortes d'informations perceptives: leur contour intonatif, leur position dans l'énoncé et leur fonction syntaxique relative au temps, à la personne, au cas, etc. Du point de vue productif, l'enfant doit apprendre les formes à utiliser pour remplir telle ou telle fonction. Dans les langues agglutinantes comme le finnois, les suffixes des cas de déclinaison semblent être très précoces. Localisés à la fin des mots, ils peuvent avoir un contenu sémantique aisément accessible. En revanche, l'acquisition des mots grammaticaux paraît tardive en finnois.

On peut noter quelques différences assez frappantes entre les énoncés à deux mots de M. dans les deux langues. En effet, les énoncés qui combinent un pronom et un verbe sont relativement courants en français, mais inexistants en finnois. Les énoncés associant une préposition et un substantif sont fréquents en français, mais très rares en finnois. Les mots de négation n'existent que dans les énoncés en français. En revanche, les énoncés qui combinent un substantif et un adjectif, ou deux substantifs sont très fréquents en finnois et très rares en français. On constate donc que les énoncés finnois contiennent surtout des mots lexicaux, les énoncés français contiennent aussi des mots grammaticaux (prépositions, pronoms, mots de négation).

8 Les énoncés hybrides confirment ces remarques. Les mots de négation français sont employés avec des substantifs finnois. Les prépositions françaises sont utilisées avec des substantifs ou des verbes en finnois. Chaque langue semble avoir ses domaines acquis rapidement et ses domaines de complexité et d'irrégularité acquis plus lentement.

Le finnois possède des adpositions (prépositions et postpositions), mais elles sont

Le finnois possède aussi un auxiliaire de négation dont l'utilisation semble être plus complexe que celle de l'adverbe de négation en français. Le verbe principal demeure invariable, c'est l'auxiliaire qui est conjugué. On a quelque chose qui ressemble aux constructions : minä en syö « je nie manger », sinä et syö « tu nies manger », hän ei syö « il 
nie manger ", etc. On comprend donc pourquoi ces formes apparaissent relativement tard dans les énoncés produits pas $M$.

71 On comprend également la rareté des pronoms personnels dans les énoncés finnois de M. En effet, en finnois le pronom personnel n'est pas nécessaire pour reconnaître la personne du verbe (sauf à la troisième personne) : laulan, «je chante ", laulat «tu chantes ", hän laulaa "il/elle chante", laulamme «nous chantons", laulatte "vous chantez », he laulavat « ils/elles chantent ».

Cette étude porte sur les énoncés d'une seule enfant. Il serait nécessaire d'étendre les observations sur d'autres enfants bilingues français-finnois, et peut-être sur d'autres langues agglutinantes ainsi que sur d'autres langues casuelles.

\section{BIBLIOGRAPHIE}

BERTONCINI, J. ; DE BOYSSON-BARDIES, B. (2003) La perception et la production de la parole avant deux ans, in Kail M. ; Fayol M. (éds) L'acquisition du langage : le langage en émergence, de la naissance à trois ans, Paris : PUF.

DEPREZ, Ch. (1994) Les enfants bilingues : langues et familles, Paris : Didier.

FERGUSON, C.A. (1986) Discovering sound units and constructing sound systems: it's child's play, in Perkell, J. S.; Klatt D.H. (eds) Invariance and variability in speech processes. Hillsdale, NJ: Lawrence Erlbaum Associates.

GENESEE, F.; NICOLADIS E. \& PARADIS J. (1995) Language differentiation in early bilingual development, Journal of Child Language, 22, 3, p. 611-631.

HOLlowKa, S.; BROSSEAU-LAPRE, F. \& PETITTO, L.A. (2002) Semantic and conceptual knowledge underlying bilingual babies' first signs and words, Language Learning, 55, p. 205-262.

JOHNSON, J.S. \& NEWPORT, E. L. (1989) Critical period effects in second language learning: the influence of maturational state on the acquisition of English as a second language, Cognitive Psychology, 21, p. 60-99.

KIM, K.H.S.; RELKIN, N. R.; LEE, K.-M. \& HIRSCH, J. (1997) Distinct cortical reas associates with native and second languages, Nature, 388, p. 171-174.

LENNEBERG, E.H. (1967) Bological foundations of language, New York: Wiley.

LEOPOLD, W. (1949) Speech Development of a Bilingual Child: A Linguist's Record, Grammar and General Problems, 3. Evanston: Northwestern University Press.

LÜDI, G. \& PY, B. (2003) Être bilingue, Bern : Peter Lang.

MACKEN, M.A. (1979) Developmental reorganization of phonology, Lingua, 49, p. 11-49.

PALLIER, Ch. \& ARGENTI, A.-M. (2003) Imagerie cérébrale du bilinguisme, in Etard O. \& TzourioMazoyer N. (éds) Cerveau et Langage. Traité de Sciences Cognitives, Paris : Hermès Sciences, p. 183-198. 
PEARSON, B.Z.; FERNANDEZ, S.C. \& OLIER, D.K. (1993) Lexical development in bilingual infants and toddlers : comparison to monolingual norms, Language Learning, 43, 1, p. 93-120.

TOIVANEN, J. (1993) Lasten erilaisuus Suomen kielen morfologian omaksunnassa, in Iivonen, A. ; Lieko, A. \& Korpilahti, P. (eds) Lapsen normaali ja poikkeava kielen kehitys, Helsinki : SKST, 583, p. 268-305.

VIHMAN, M. (1985) Language differentiation by the bilingual infant, Journal of Child Language, 12, p. 297-324.

\section{NOTES}

1. Le bilinguisme précoce peut également être consécutif. Dans ce cas-là, l'enfant commence à être en contact avec la seconde langue après l'âge de 3 ans.

2. Les chiffres entre crochets indiquent l'âge de l'enfant au moment où elle a commencé à utiliser le mot, ex. : 1,6 signifie un an et six mois.

\section{RÉSUMÉS}

Cette étude porte sur les premiers énoncés produits par une enfant bilingue précoce francophone-finnophone. Tout d'abord, comme ses énoncés à un terme contiennent soit un mot français, soit un mot finnois, il est intéressant d'examiner la nature de ces mots et de souligner les similitudes et les différences au niveau des deux lexiques. Ensuite, parmi ses énoncés à deux termes, on trouve des combinaisons de deux mots en français et des combinaisons de deux mots en finnois ainsi que des assemblages hybrides qui combinent un mot français et un mot finnois. On peut s'intéresser à la composition grammaticale et sémantique de ces énoncés pour mettre en relief les particularités constatées dans les deux langues. Enfin, l'étude des énoncés hybrides permet de découvrir la nature des premiers mélanges linguistiques.

Il s'agit d'un parcours d'observation et de réflexion à travers les pratiques bilingues d'une enfant qui ancre la démarche dans un contexte authentique. Le fait d'examiner le langage d'un seul enfant autorise à avancer modestement quelques hypothèses, mais interdit, bien évidemment, la généralisation des résultats.

The aim of this is article is to analyse the first sentences produced by a simultaneous bilingual French-Finnish child. Her one word-sentences contain one word in French or one word in Finnish. It is interesting to examine the nature of these words and to discover the similarities and the differences in her two lexicons. Her two words-sentences can combine two French words or two Finnish words. We can also find hybrid sentences associating a French word and a Finnish word. It is important to observe the grammatical and semantic composition of these sentences and to accentuate the particularities noticed in both languages. The hybrid sentences help us to discover the nature of the first linguistic mixtures.

We observe the bilingual practices of only one child in an authentic context. That's why we can advance modestly some hypotheses, but we can't generalize the results. 
INDEX

Mots-clés : acquisition, bilinguisme, lexicologie, sémantique, contact de langues

Keywords : acquisition, bilingualism, lexicology, semantic, language contact

\section{AUTEUR}

\section{AIINO NIKLAS-SALMINEN}

Laboratoire Parole et Langage, CNRS, Aix-Marseille Université

aino.niklas-salminen@lpl-aix.fr 